\title{
Simulação da evolução temporal de uma pluma de contaminação em área de disposição de resíduos sólidos urbanos (RSUs)
}

\author{
Ferreira, D.A. *, IAG-USP; Ustra, A.T., IAG-USP; Elis, V.R., IAG-USP
}

Copyright 2018, SBGf - Sociedade Brasileira de Geofísica

Este texto foi preparado para a apresentação no VIII Simpósio Brasileiro de Geofísica, Salinópolis, 18 a 20 de setembro de 2018 . Seu conteúdo foi revisado pelo Comitê Salinópolis, 18 a 20 de setembro de 2018. Seu conteúdo foi revisado pelo Comitê
Técnico do VIII SimBGt, mas não necessariamente representa a opinião da SBGf ou de seus associados. É proibida a reprodução total ou parcial deste material para propósitos comerciais sem prévia autorização da SBGf.

\begin{abstract}
Contamination plumes generally contain a high amount of dissolved salts and therefore define geophysical signatures of high electrical conductivity, which makes geoelectrical methods suitable for the study of contaminated areas. This work presents the preliminary results of a research project whose objective is to simulate geophysical monitoring of a contamination plume generated by a waste disposal area. The obtained models will compose a study about the effects of the inhibition of biodegradation in the electrical response in lysimeters constructed by Faculty of Architecture and Civil Engineering - University of Campinas (FEC-UNICAMP). The results presented in this paper suggest that geophysical monitoring can evaluate the temporal and spatial evolution of a plume of contamination.
\end{abstract}

\section{Introdução}

A crescente geração de resíduos sólidos domésticos, industriais e hospitalares, associada à fatores socioeconômicos e demográficos, constitui uma das maiores preocupações da população urbana. O manejo e o descarte inadequados desses materiais estão diretamente relacionados à problemas ambientais e de saúde pública. Entre os danos decorrentes do mau gerenciamento dos resíduos sólidos urbanos (RSUs) destaca-se a contaminação das águas subterrâneas, que ocorre quando o chorume (formado pela percolação da água das chuvas no corpo de resíduos e pela degradação destes) se infiltra no solo, atingindo a zona saturada e espalhando contaminantes por grandes áreas (Ustra, 2008).

As plumas de contaminação que resultam desse processo apresentam alto teor de sais dissolvidos e definem, portanto, assinaturas geofísicas de baixa resistividade elétrica (Braga, 2001), que tornam os métodos geoelétricos (da eletrorresistividade e da polarização induzida) apropriados para a caracterização de áreas de disposição de RSUs (Ustra, 2008). Além disso, a geofísica também pode ser empregada no monitoramento da pluma de contaminação. Isto porque a repetição dos levantamentos geofísicos na área contaminada permite identificar mudanças temporais das assinaturas geofísicas relacionadas à contaminação (Ustra, 2017).
O objetivo deste estudo é justamente simular o monitoramento geofísico da pluma de contaminação de uma área de disposição de RSUs, assumida como um meio isotrópico saturado no qual um soluto (cloreto de sódio, $\mathrm{NaCl}$ ) é injetado instantaneamente ou continuamente (Feitosa \& Filho, 2000). Os modelos obtidos passarão a compor um estudo acerca dos efeitos da inibição da biodegradação na resposta elétrica de lisímetros construídos pela Faculdade de Engenharia Civil, Arquitetura e Urbanismo da Universidade de Campinas (FEC - UNICAMP). Os lisímetros são modelos físicos experimentais que simulam as transformações biológicas e geomecânicas que atuam nas áreas de disposição de RSUs (Almeida et al., 2015). Espera-se que o monitoramento geofísico (time-lapse) seja capaz de detectar variações de resistividade decorrentes de mudanças do estado da pluma de contaminação.

\section{Teoria e Método}

A primeira etapa da simulação consistiu em determinar a distribuição da concentração do soluto (em ppm) a partir do ponto de injeção (instantânea $\mathrm{C}_{\|}$ou contínua $\mathrm{C}_{\mathrm{IC}}$ ) para diferentes instantes. As soluções apresentadas por Feitosa \& Filho (2000) para as duas formas de injeção de soluto são expressas pelas equações abaixo:

$$
\begin{gathered}
C_{I I}(x, t)=\frac{C_{0} / \phi}{\sqrt{4 \pi D_{x} t}} \exp \left[\frac{-\left(x-x_{0}\right)^{2}}{4 D_{x} t}\right] \\
C_{I C}(x, t)=\frac{C_{0}}{2}\left[\operatorname{erfc}\left(\frac{x-x_{0}}{\sqrt{2} \sigma}\right)+\exp \left(\frac{v x}{D_{x}}\right) \operatorname{erfc}\left(\frac{x+x_{0}}{\sqrt{2} \sigma}\right)\right]
\end{gathered}
$$

onde $C_{0}$ é a concentração do soluto no volume injetado (em ppm), $\phi$ é a porosidade, $x_{0}=v t, \sigma=\sqrt{2 D_{x} t}, v$ é a velocidade média de advecção do soluto $(\mathrm{em} \mathrm{m} / \mathrm{s})$ e $D_{x}$ é o coeficiente de dispersão (em $\left.\mathrm{m}^{2} / \mathrm{s}\right)$.

As concentrações obtidas foram então convertidas em condutividade elétrica por meio da expressão $\sigma_{e}=0,64 *$ $C(x, t)$ (Lenntech, 2018). Segundo Weller \& Slater (2012), a maioria dos modelos para a condutividade elétrica complexa de um material poroso em baixas frequências (isto é, menores que $100 \mathrm{~Hz}$ ) são baseados na adição de dois termos de condução:

$$
\sigma^{*}=\sigma_{n}+\sigma_{s}^{*}
$$

onde $\sigma_{n}$ é a condutividade normal e $\sigma_{s}^{*}$ é a condutividade superficial complexa.

A condutividade normal representa a contribuição da condução eletrolítica que ocorre no espaço entre poros interligados e pode ser expressa como (Ward, 1990):

$$
\sigma_{n}=\sigma_{e} / F
$$


onde $\sigma_{e}$ é a condutividade do eletrólito e $F$ é o fator de formação, cujo valor é fornecido pela forma geral da Lei de Archie.

A condutividade superficial complexa representa o efeito dos minerais de argila na condutividade total da rocha (Ward, 1990). A alta condutividade dos minerais de argila dá origem a uma dupla camada elétrica, composta pela camada fixa ou de Stern e pela camada difusa. A contribuição dessa dupla camada elétrica para o caso de partículas de caulinita saturadas por uma solução de $\mathrm{NaCl}$ é dada por (Leroy \& Revil, 2009):

$$
\sigma_{s}^{*}=\frac{2}{a_{0}}\left(\sum_{s}^{0}+\sum_{s}^{\infty}\right)-\frac{2}{a_{0}} \frac{\sum_{s}^{\infty}}{\left(1+i \omega \tau_{0}\right)}
$$

onde $\sum_{s}^{\infty}=\beta_{i}^{S} e \Gamma_{i}^{0}$ representa a contribuição da camada de Stern em altas frequências, $\sum_{s}^{0}=\beta_{i} e \Gamma_{i}^{d}$ representa a contribuição da camada difusa, $a_{0}$ é o raio do grão (em metros), $\tau_{0}$ é o tempo de relaxação e e é a carga elementar (tomada como positiva, $e=1.6 \times 10^{-19} \mathrm{C}$ ).

A condutividade elétrica complexa pode ser medida como a magnitude e a diferença de fase entre a corrente elétrica aplicada e a voltagem observada (domínio da frequência). A extensão de uma medida no domínio da frequência para medidas em uma faixa de frequências constitui o método IP espectral. A condutividade real $(\sigma)$ representa as correntes de condução ôhmica (perda de energia), enquanto a condutividade imaginária $\left(\sigma^{\prime \prime}\right)$ representa, em baixas frequências, o termo de polarização (armazenamento de energia). A diferença de fase $(\varphi)$ é análoga ao parâmetro cargabilidade $(m)$, determinado pelas medidas do efeito IP no domínio do tempo.

Os modelos foram desenvolvidos utilizando-se o software MATLAB R2015a e os dados inseridos no modelo final provêm de Feitosa \& Filho (2000) e Leroy et al. (2008).

\section{Resultados}

A distribuição da concentração de soluto a partir dos pontos de injeção instantânea e contínua foi calculada a partir das equações 1 e 2 , respectivamente, utilizando os parâmetros fornecidos na Tabela 1.

Tabela 1 - Parâmetros de entrada para os modelos de transporte de contaminantes

\begin{tabular}{c|c|c}
\hline Parâmetro & Valor & Referência \\
\hline $\mathrm{C}_{0}$ & $\begin{array}{c}4.0 \times 10^{-4} \mathrm{~mol} / \mathrm{L} \\
(23.4 \mathrm{ppm})\end{array}$ & $\begin{array}{c}\text { Leroy \& Revil } \\
(2008)\end{array}$ \\
\hline$\phi$ & 0.4 & $\begin{array}{c}\text { Feitosa \& Filho } \\
(2000)\end{array}$ \\
\hline $\mathrm{D}_{\mathrm{x}}$ & $5.0 \times 10^{-11} \mathrm{~m}^{2} / \mathrm{s}$ & $\begin{array}{c}\text { Feitosa \& Filho } \\
(2000)\end{array}$ \\
\hline $\mathrm{v}$ & $2.5 \times 10^{-7} \mathrm{~m} / \mathrm{s}$ & $\begin{array}{c}\text { Feitosa \& Filho } \\
(2000)\end{array}$ \\
\hline
\end{tabular}

O modelo de injeção instantânea é mostrado na Figura 1. O pico da concentração do soluto se encontra no ponto
(0 m, 13750 ppm) após $8 \mathrm{~h}$ da injeção e no ponto $(0 \mathrm{~m}$, 7918 ppm) após $24 \mathrm{~h}$ da injeção. Para o modelo de injeção contínua, mostrado na Figura 2, o pico da concentração do soluto se encontra no ponto $(0.0025 \mathrm{~m}$, $23.4 \mathrm{ppm})$ após $8 \mathrm{~h}$ da injeção e no ponto $(0.01 \mathrm{~m}, 23.4$ ppm) após 24 h da injeção.

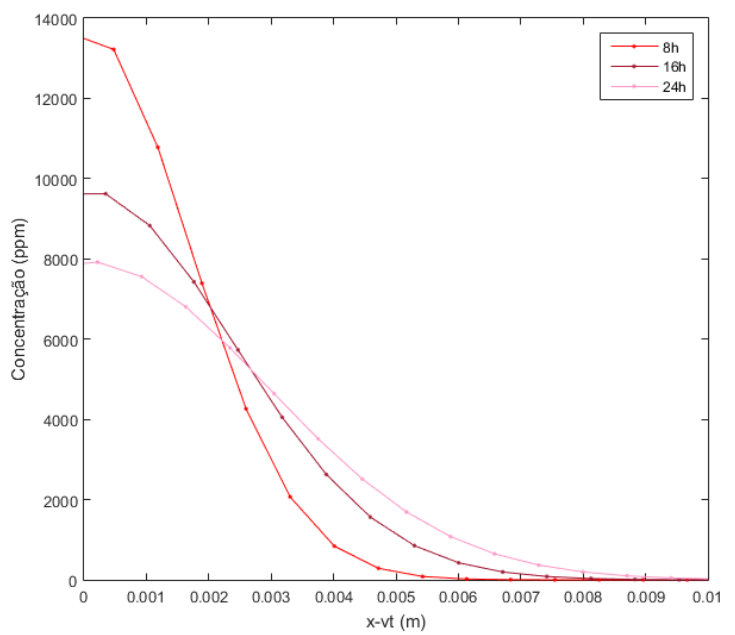

Figura 1 - Distribuição da concentração de um soluto injetado instantaneamente.

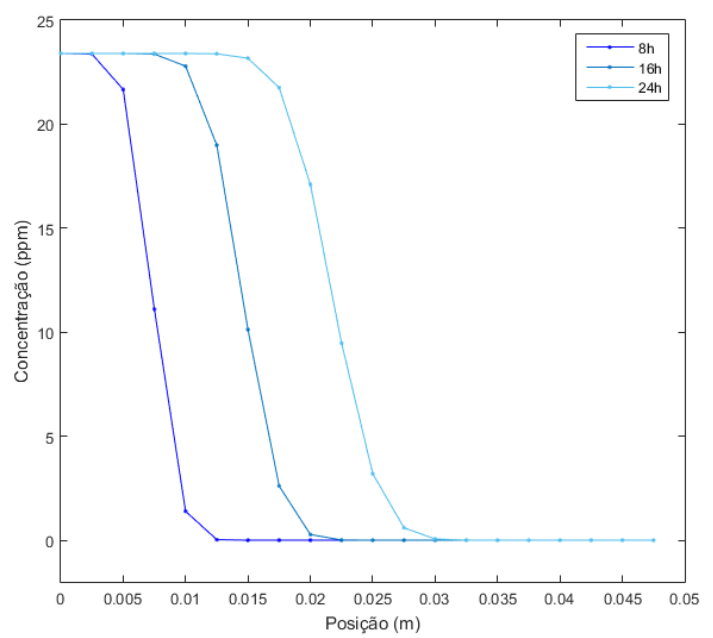

Figura 2 - Distribuição da concentração de um soluto injetado continuamente.

O modelo de condutividade elétrica complexa em função da frequência utilizou os parâmetros fornecidos na Tabela 2. As Figuras 3 e 4 apresentam os resultados obtidos para os modelos de injeção instantânea e contínua, respectivamente. O componente real da condutividade elétrica complexa reflete a contribuição dominante do eletrólito, isto é, da pluma de contaminação, enquanto o componente imaginário reflete as propriedades superficiais dos grãos minerais 
presentes no meio poroso. A fase, por sua vez, contabiliza os dois efeitos simultaneamente.

Tabela 2 - Parâmetros de entrada para o modelo de caulinita saturada por uma solução de $\mathrm{NaCl}$

\begin{tabular}{c|c|c}
\hline Parâmetro & Valor & Referência \\
\hline $\mathrm{C}_{0}$ & $\begin{array}{c}4 \times 10^{-4} \mathrm{~mol} / \mathrm{L} \\
(23.4 \mathrm{ppm})\end{array}$ & $\begin{array}{c}\text { Leroy \& Revil } \\
(2008)\end{array}$ \\
\hline $\mathrm{a}$ & 0.8 & Estimado \\
\hline $\mathrm{m}$ & 1.8 & Estimado \\
\hline$\phi$ & 0.4 & $\begin{array}{c}\text { Feitosa \& Filho } \\
(2000)\end{array}$ \\
\hline$\beta_{\mathrm{i}}^{\mathrm{s}}$ & $5.19 \times 10^{-8} \mathrm{~m}^{2} / \mathrm{sV}$ & $\begin{array}{c}\text { Leroy \& Revil } \\
(2008)\end{array}$ \\
\hline$\beta_{\mathrm{i}}$ & $5.19 \times 10^{-8} \mathrm{~m}^{2} / \mathrm{sV}$ & Estimado \\
\hline$\Gamma_{\mathrm{i}}^{0}$ & $6.5 \times 10^{16} 1 / \mathrm{m}^{2}$ & $\begin{array}{c}\text { Leroy \& Revil } \\
(2008)\end{array}$ \\
\hline$\Gamma_{\mathrm{i}}^{\mathrm{d}}$ & $7.5 \times 10^{16} 1 / \mathrm{m}^{2}$ & $\begin{array}{c}\text { Leroy \& Revil } \\
(2008)\end{array}$ \\
\hline $\mathrm{a}_{0}$ & $3.5 \times 10^{-5} \mathrm{~m}$ & $\begin{array}{c}\text { Leroy \& Revil } \\
(2008)\end{array}$ \\
\hline
\end{tabular}

Nas Figuras 3 e 4, nota-se que o componente imaginário da condutividade elétrica complexa não sofre variação durante as $24 \mathrm{~h}$ que sucedem a injeção do soluto. No entanto, percebe-se que a fase e o componente real variam durante esse intervalo de tempo. No modelo de injeção instantânea (Figura 3), a fase aumenta com o tempo, enquanto o componente real diminui. No modelo de injeção contínua (Figura 4), a fase diminui com o tempo e o componente real aumenta.
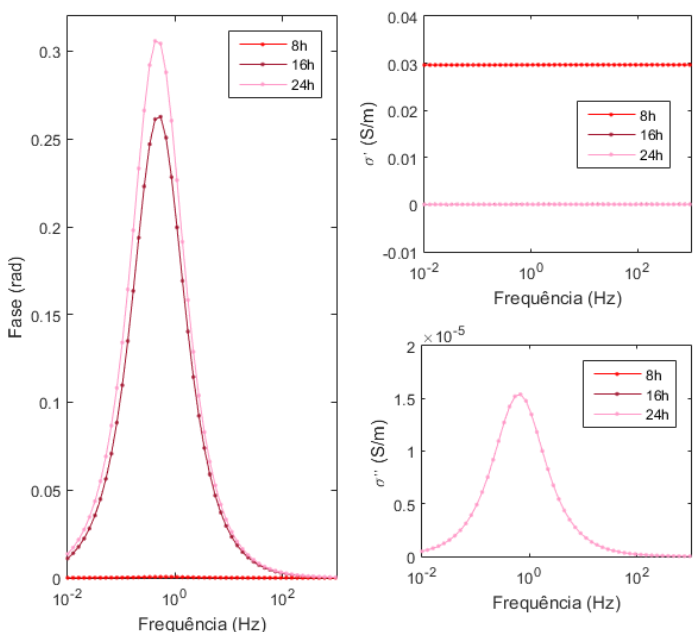

Figura 3 - Condutividade complexa em função da frequência para o modelo de injeção instantânea.
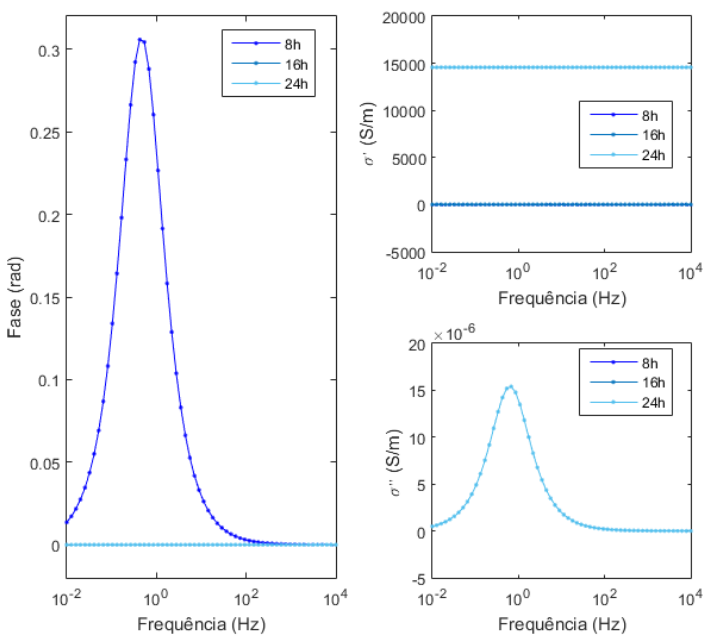

Figura 4 - Condutividade complexa em função da frequência para o modelo de injeção contínua.

A evolução temporal da condutividade elétrica complexa nos pontos de pico da frequência está representada nas Figuras 5 e 6 abaixo. Observa-se, em ambos os modelos de injeção de soluto, que o componente real e a fase da condutividade elétrica complexa sofrem variação temporal e espacial, enquanto o componente imaginário permanece inalterado.
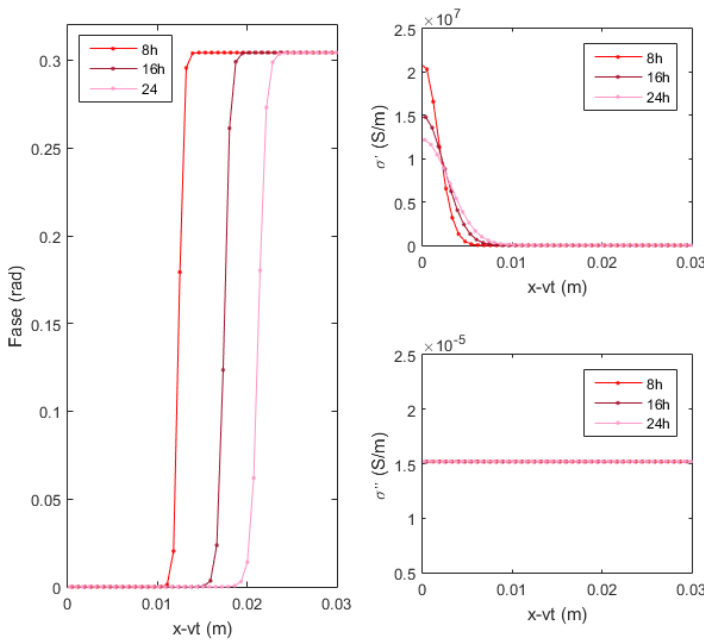

Figura 5 - Evolução temporal da condutividade complexa nos pontos de pico da frequência para o modelo de injeção instantânea. 

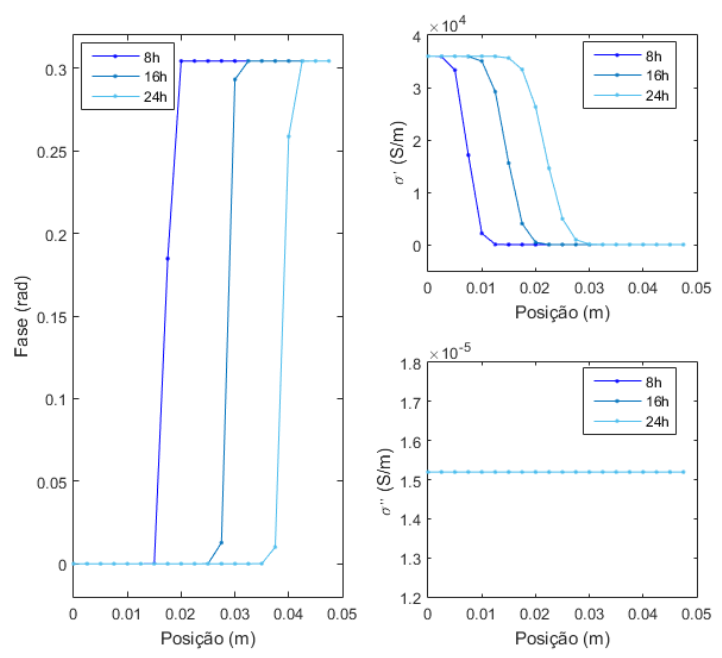

Figura 6 - Evolução temporal da condutividade complexa nos pontos de pico da frequência para o modelo de injeção contínua.

\section{Discussão e Conclusões}

A partir do instante em que o soluto é injetado, tem início a formação de uma zona de variação progressiva da concentração (Feitosa \& Filho, 2000). Esse padrão pode ser observado na distribuição da concentração de soluto nos casos de injeção instantânea ou contínua (Figuras 1 e 2). Quando a injeção de soluto não é regular (Figura 1), verifica-se que a largura da zona de transição entre os valores da concentração tende a ampliar-se com o tempo. Também é possível notar que o soluto se espalha na direção do fluxo e na direção normal ao mesmo. Quando a injeção é contínua (Figura 2), no entanto, a largura da zona de transição não varia com tempo e o soluto se espalha apenas na direção do fluxo. Em ambos os casos, a distribuição da concentração à medida que o soluto se move no meio poroso é alterada pelos fenômenos da advecção e da dispersão hidrodinâmica.

A simulação do comportamento espectral da condutividade elétrica (Figuras 3 e 4 ) revela os efeitos do eletrólito e do meio simulado. Os valores de pico da fase e do componente imaginário relacionam-se à granulometria simulada.

Para cada posição na pluma de contaminação, o valores dos picos de frequência foram plotados (Figuras 5 e 6). Enquanto a parte real da condutividade reflete as mudanças da pluma de contaminação (que sofre dispersão), a parte imaginária permanece inalterada, como era esperado, uma vez que nenhuma transformação do meio poroso foi simulada.

Os resultados apresentados nesse trabalho fazem parte de um projeto em andamento. Até o momento, as simulações mostram que o monitoramento geofísico com os métodos eletrorresistividade e polarização induzida pode avaliar a evolução temporal e espacial de uma pluma de contaminação. Em casos reais, a integração dos dois métodos permite avaliar variações temporais relativas à mudança na composição do fluido do poros e do meio.

As etapas futuras consistem em simular diferentes taxas de variação da concentração de contaminantes e seu efeito nos dados de condutividade elétrica. Espera-se com isso, avaliar a sensibilidade da condutividade elétrica complexa (eletrorresistividade e polarização induzida) a diferentes estágios de degradação dos resíduos que formam a pluma de contaminação.

\section{Referências}

Almeida, G. H. T. D.; Manzatto, M. P.; Favery, R. L. T; Moretto, R. L.; Teixeira, E. N.; Miguel, M. G.. Construção de lisímetros para estudo do comportamento geomecânico dos resíduos sólidos urbanos. In: Congresso Brasileiro de Geologia de Engenharia e Ambiental - CBGE, 2015, Bento Gonçalves - RS. Congresso Brasileiro de Geologia de Engenharia e Ambiental - CBGE, 2015. v. 1.

Braga, A.C.O., 2001. Métodos Geoelétricos Aplicados: Módulo Hidrogeologia. Universidade Estadual Paulista UNESP/IGCE/DGA. Rio Claro SP. 91p..

Feitosa, F.A.C. \& Filho, J.M., 2000. Hidrogeologia: Conceitos e Aplicações. 2.ed. CPRM/LABHID, 391 p.

Lenntech, 2018. Disponível em: < https://www.lenntech.com/calculators/conductivity/tds_en gels.htm > . Acessado em 28 de março de 2018.

Leroy, P., Revil, A., Kemna, A., Cosenza, P. \& Ghorbani, A., 2008. Complex conductivity of water-saturated packs of glass beads. Journal of Colloid and Interface Science, Vol. 321: 103-117.

Leroy, P. \& Revil, A., 2009. A mechanistic model for the spectral induced polarization of clay materials. Journal of Geophysical Research, Vol. 114, B10202.

Ustra, A.T., 2008. Utilização dos métodos eletrorresistividade e polarização induzida com aquisição de dados 3D para caracterização geoambiental de uma área à jusante do aterro de resíduos sólidos urbanos de Bauru - SP. Dissertação (Mestrado em Geofísica), Instituto de Astronomia, Geofísica e Ciências Atmosféricas da Universidade de São Paulo, São Paulo. $182 \mathrm{p}$.

Ustra, A.T., 2017. Geofísica Ambiental. Apostila Interna da Escola de Verão de Geofísica, Instituto de Astronomia, Geofísica e Ciências Atmosféricas da Universidade de São Paulo, São Paulo.

Ward, S.H., 1990. Resistivity and Induced Polarization Methods. Geotechnical and Environmental Geophysics, Volume 1: Review and Tutorial. Society of Exploration Geophysics, Tulsa, OK, pp. 147-189.

Weller, A. \& Slater, L., 2012. Salinity dependence of complex conductivity of unconsolidated and consolidated materials: Comparisons with electrical double layer models. Geophysics, Vol. 77, no. 6, D185-D198. 